Classification

Physics Abstracts

$61.70 \mathrm{~J}-61.70 \mathrm{P}-62.20 \mathrm{~F}$

\title{
Transmission electron microscopy of semi-insulating Ga As deformed at room temperature and under confining pressure
}

\author{
A. Lefebvre, P. François and J. di Persio \\ Laboratoire de Structure et Propriétés de l'Etat Solide (L.A. 234), \\ Université des Sciences et Techniques de Lille, 59655 Villeneuve d'Ascq Cedex, France
}

(Reçu le 28 mai 1985, accepté sous forme définitive le 17 septembre 1985)

\begin{abstract}
Résumé. - Des échantillons de Ga As sont déformés à température ambiante et sous une pression de confinement de $600 \mathrm{MPa}$. Deux mécanismes de déformation plastique sont proposés. Le premier s'appuie sur l'observation de dislocations $\alpha$ parfaites ou peu dissociées très mobiles. Le second mécanisme fait intervenir des dislocations $\beta$ (ou vis) largement dissociées et moins mobiles. La dissociation de ces dislocations pourrait être due à une mobilité plus faible des dislocations partielles $30^{\circ}(\beta)$.

Abstract. - Ga As single crystals have been deformed by compression at room temperature under a confining pressure of $600 \mathrm{MPa}$. Two competitive mechanisms of plastic deformation are proposed. The first mechanism is supported by the observation of mobile perfect (or slightly dissociated) $\alpha$ dislocations. The second mechanism involves less mobile widely dissociated $\beta$ (or screw) dislocations. It is suggested that the large dissociation of these dislocations is due to the lower mobility of the $30^{\circ}(\beta)$ partials.
\end{abstract}

\section{Introduction.}

The macroscopic plastic behaviour of $\mathrm{Ga}$ As has been studied during the past twenty years by creep [1] and by deformation at constant strain rate (see for instance [2] to [5]; these studies have been restricted to temperatures above $250^{\circ} \mathrm{C}\left(0.35 T_{\mathrm{m}}\right)$ owing to the brittle behaviour of this material at lower temperatures.

Although only a few transmission electron microscopy (TEM) observations of the microstructures induced by deformation in III-V compounds have been made so far, there is an increasing evidence that, like in silicon and germanium, the moving dislocations are dissociated. It is well-known that, when conducting deformation tests in the high temperature range, a way to preserve the dissociation of the under-stress moving dislocations is to freeze the configurations by lowering temperature fast and applying a high enough stress on the sample [6]. Another way is to perform tests at low temperatures, i.e. with very high stresses; it has been suggested that in such conditions, the deformation could result from the propagation of isolated partials [7]. Slip by dislocation glide has been obtained very recently by microindentation of $\mathrm{Ga}$ As at room temperature $\left(0.19 T_{\mathrm{m}}\right)[8,9]$. The brittle behaviour can also be avoided with a confining pressure as has been shown in compression tests on silicon $[10,11]$. We have applied this technique to 
$\mathrm{Ga}$ As, with the view of studying the high stress deformation mechanisms in this material. The present paper reports on a preliminary TEM investigation of the defects found in the corresponding samples.

\section{Experimental results.}

2.1 Mechanical testing. - The samples for the uniaxial compression tests were cut from a Cr-doped semi-insulating Ga As crystal (supplied by LCR Thomson, Orsay, France) in the shape of rectangular bars (the specimen size was about $3.3 \times 3.3 \times 10 \mathrm{~mm}^{3}$ ); the compression axis was along [312]. The lateral faces were of the $\{111\}$ and $\{154\}$ types and the (154) face was mechanically ground before testing in order to introduce surface $\alpha$ dislocation sources [12-14]. Compression tests at room temperature were performed at a constant strain rate $\left(8 \times 10^{-4} \mathrm{~s}^{-1}\right)$ under a hydrostatic pressure of $600 \mathrm{MPa}$ in a Griggs apparatus similar to the one described in [15]. The solid confining medium used as pressure transmitter was lead and the sample was jacketed with teflon. Figure 1 shows a typical stress-strain curve; it does not show the phenomena of upper yield, usually related to the mechanism of enhanced dislocation multiplication.

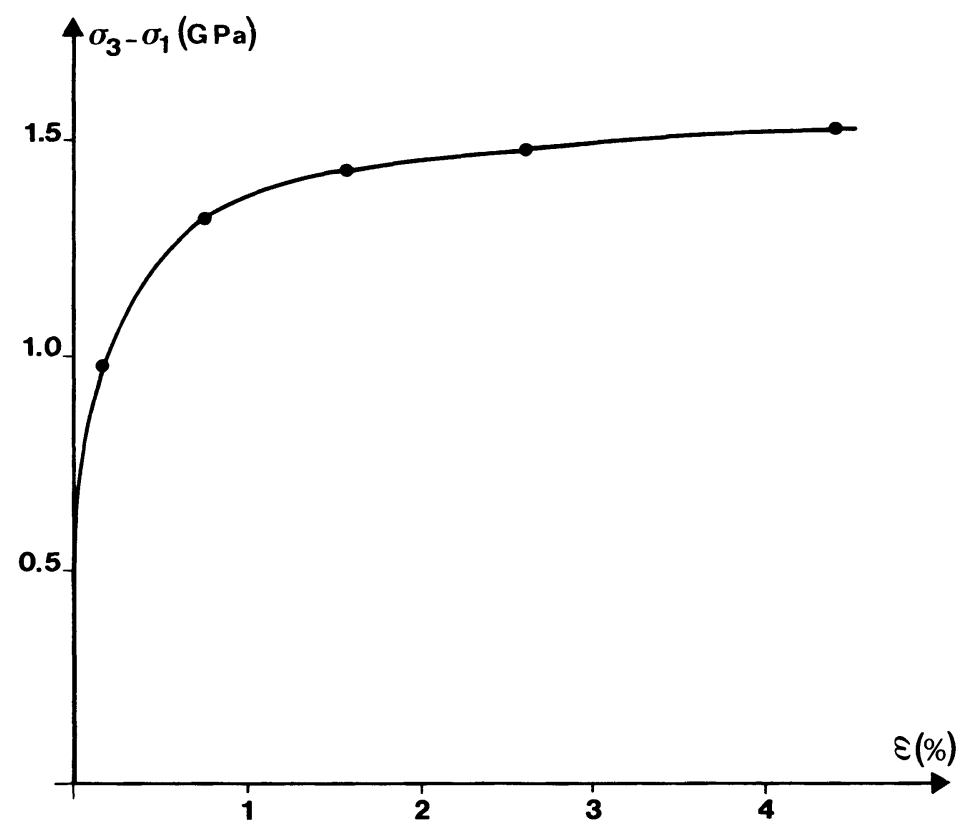

Fig. 1. - Stress-strain curve of $\mathrm{Ga}$ As deformed at room temperature under a hydrostatic pressure of $600 \mathrm{MPa} . \sigma_{3}-\sigma_{1}$ and $\varepsilon$ are unresolved stress and strain.

Slip lines are found only on the polished $(1 \overline{54})$ side face ; they correspond to the (111) $1 / 2$ [110] expected primary slip system with a $0.47 \mathrm{Schmid}$ factor. Microcraks normal to the compression axis are also observed in the deformed samples; they are probably due to stress relaxation during unloading and depressurization.

2.2 TRANSMISSION ELECTRON MiCROSCOPY. - Samples for TEM were taken from three deformed samples with $\varepsilon=4 \%$. Owing to the microcracks previously described, these samples were 
cut parallel to the $(\overline{3} 1 \overline{2})$ plane and thin specimens were prepared by mechanical thinning and ion-beam milling. TEM studies were performed with a JEOL $200 \mathrm{CX}$ operated at $200 \mathrm{kV}$.

2.2.1 (111) glide bands. - Two types of glide bands parallel to the (111) plane are observed. The first glide bands (type A) are very thin and homogeneously distributed in the thin foils (Fig. 2). They contain dislocations of the primary slip system with Burgers vector 1/2 [1 $\overline{10}$ ]. Most of them are left and right handed screw dislocations and the difference in the sign of their Burgers vectors can be recognized by the complementary behaviour of the fringes along the dislocation images [16]; $60^{\circ}$ dislocations are not observed and the other orientations of the dislocation lines are thought to be due to interactions between screw dislocations within the glide bands. All these dislocations do not appear to be dissociated in weak-beam conditions : if dissociated, their width is smaller than $50 \AA$ [as the dissociation plane (111) of the dislocations is steeply inclined to the foil surface, dissociation widths less than $50 \AA$ cannot be detected by the weak-beam technique). These results are compatible with the dissociation widths of screw dislocations observed at higher temperatures (see for instance [17]).

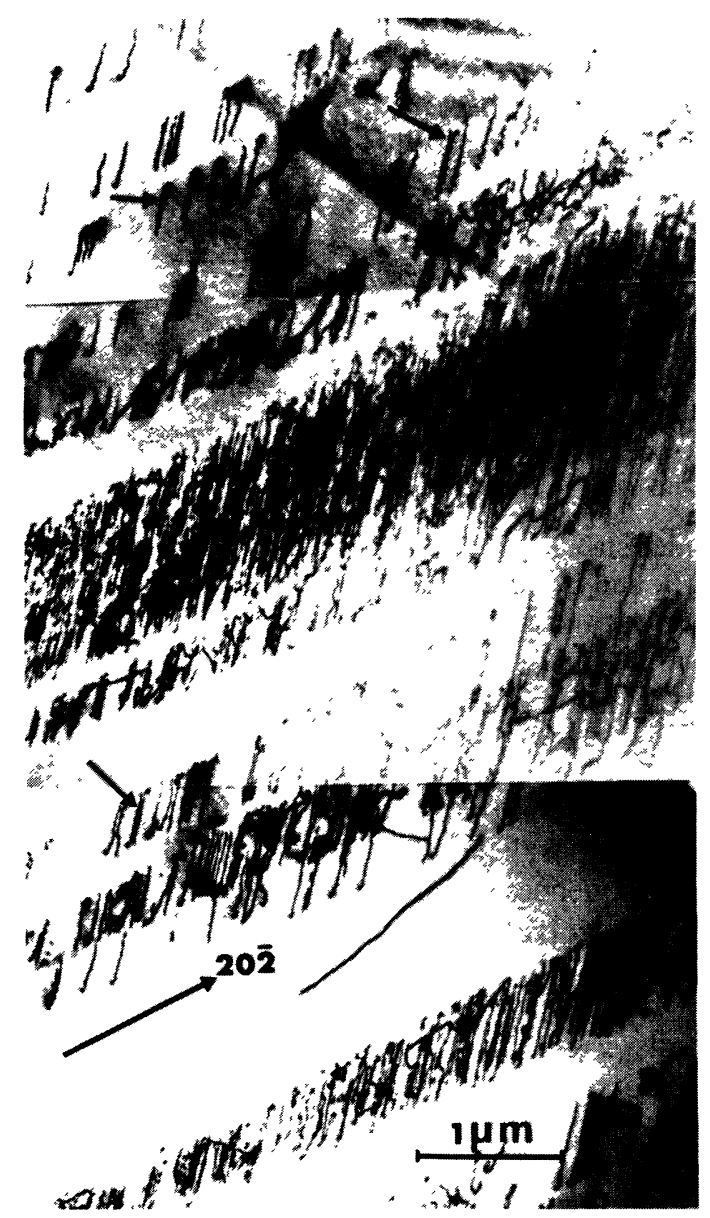

Fig. 2. - «A $॥$ glide bands of the primary slip system. Bright-field. $\mathbf{g}=(20 \overline{2})$. Some groups of screw dislocations exhibiting black-white oscillations are arrowed. 
The glide bands of the second type B are fewer and heterogeneously distributed in the samples. They contain extended stacking faults and microtwins. The stacking faults are intrinsic and left behind by the uncorrelated motion of partial dislocations with Burgers vectors $1 / 6$ [211] and $1 / 6$ [121] (Fig. 3) (Schmid factors are respectively 0.34 and 0.47 for these dislocations). Overlapping stacking faults are commonly observed and the corresponding diffraction patterns

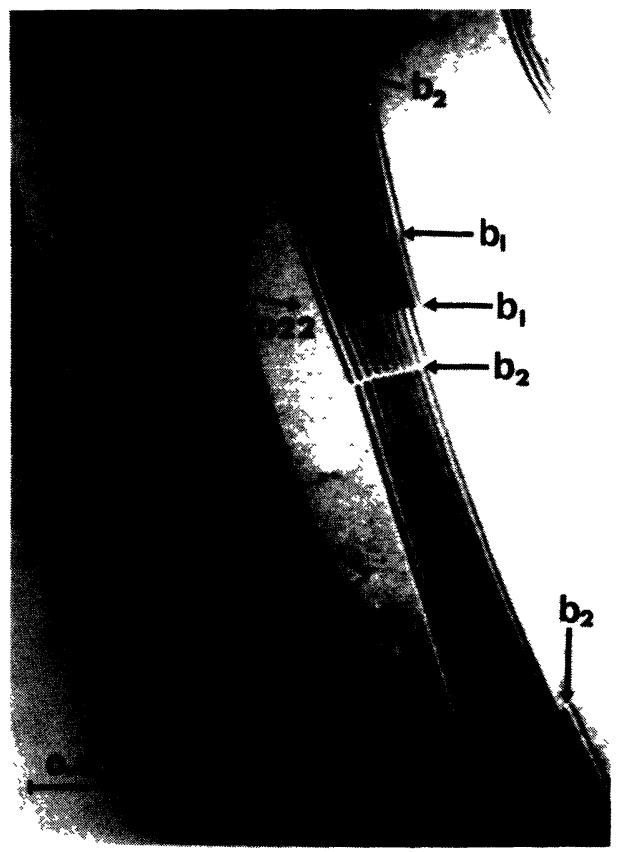

Fig. 3. - Intrinsic (111) extended stacking faults in B glide bands. Bright-field. $g=(022)$. a : single stacking

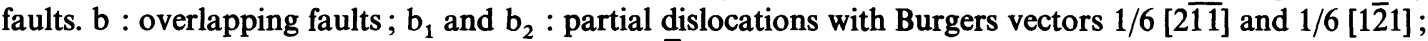
the other dislocation have Burgers vector $1 / 2[1 \overline{1} 0]$.

show continuous streaking parallel to the reciprocal direction [111]*. When the diffraction patterns taken in beam directions parallel to the faulted plane show well-defined intensity maxima on the streaks, twin stacking sequences can be identified [18] : figure 4 shows an area containing such (111) twin nuclei and the corresponding $(\overline{110})^{*}$ diffraction pattern.

2.2 .2 (111) microtwins. - The width of these twins never exceeds $\sim 300 \AA$ and they are never found isolated in the thin foils. Figure 5 shows the overlapping of four twin domains : two types of twinning dislocations are found in the (111) matrix - twin interfaces. Their Burgers vectors are $b_{1}=1 / 6$ [21 $\left.\overline{1}\right]$ and $b_{2}=1 / 6$ [121] (Schmid factors 0.30 in both cases). These partial dislocations show preferential directions : [211] for $b_{1}$ screw dislocations, [121] and [110] for $b_{2}$ screw and $30^{\circ}$ dislocations. Contrary to (111) twins, the corresponding diffraction patterns do not show any diffuse streaking parallel to [111]* and only additional diffraction spots characteristic of the twin domains are observed; so the (111) twin domains contain no stacking faults as can be also found by bright and dark-field TEM observations. 

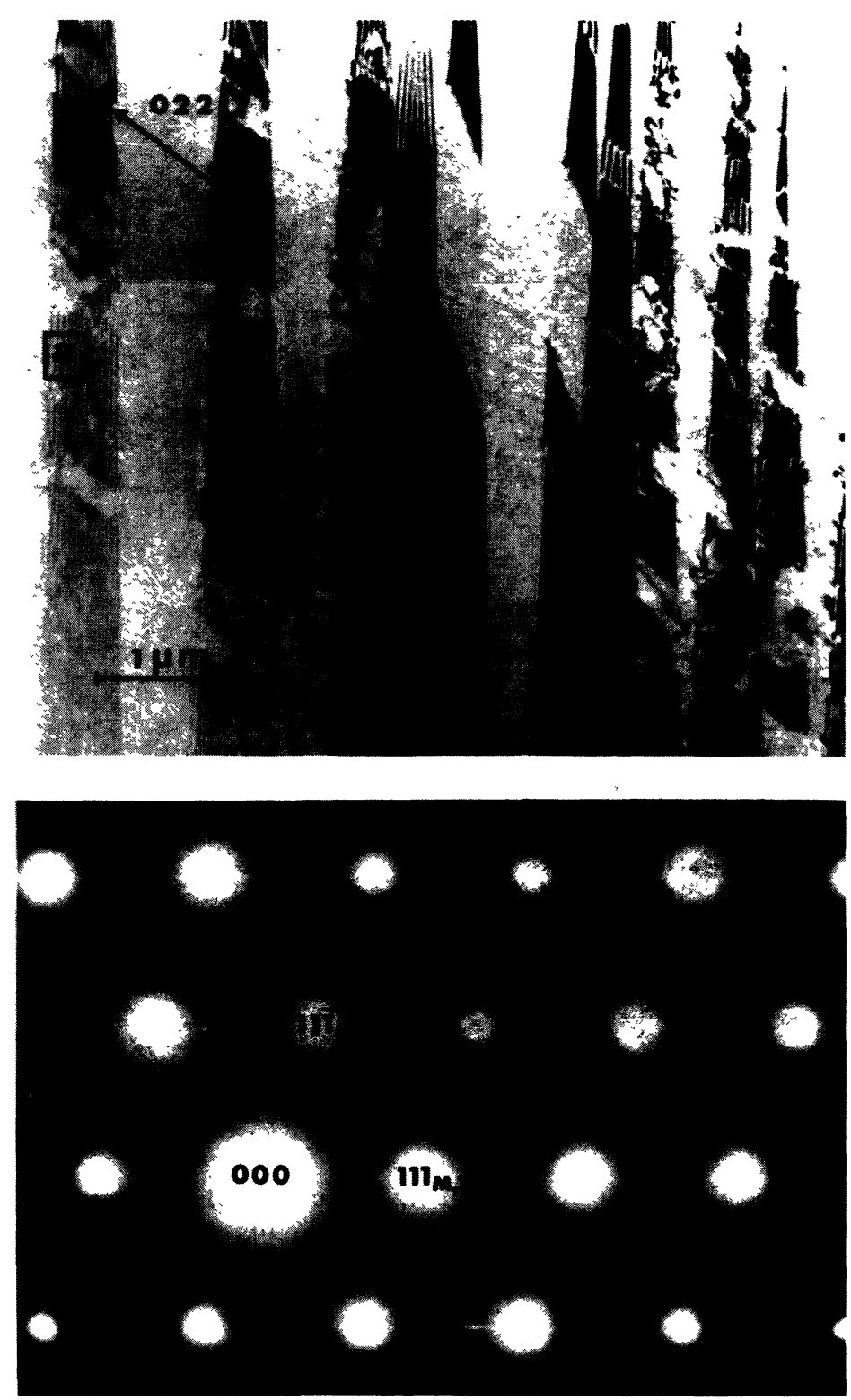

Fig. 4. - (111) microtwinning in B glide bands. a : Bright-field. $\mathbf{g}=(022)$. Regions in which the number of faulted planes is a multiple of three show a faint or no fringe contrast $[19] . b:[1 \overline{1} 0]_{M}=[1 \overline{1} 0]_{T}$ diffraction pattern; $M$ and $T$ subscripts respectively refer to matrix and twin.

\section{Discussion.}

The creation of two types of (111) $1 / 2$ [110] glide bands is the main characteristic of the plastic deformation of $\mathrm{Ga}$ As at room temperature. The A glide bands contain perfect (or slightly dissociated) dislocations; the B glide bands are much fewer and contain widely dissociated dislocations and microtwins. Other preliminary TEM observations of the A glide bands in (111) thin 

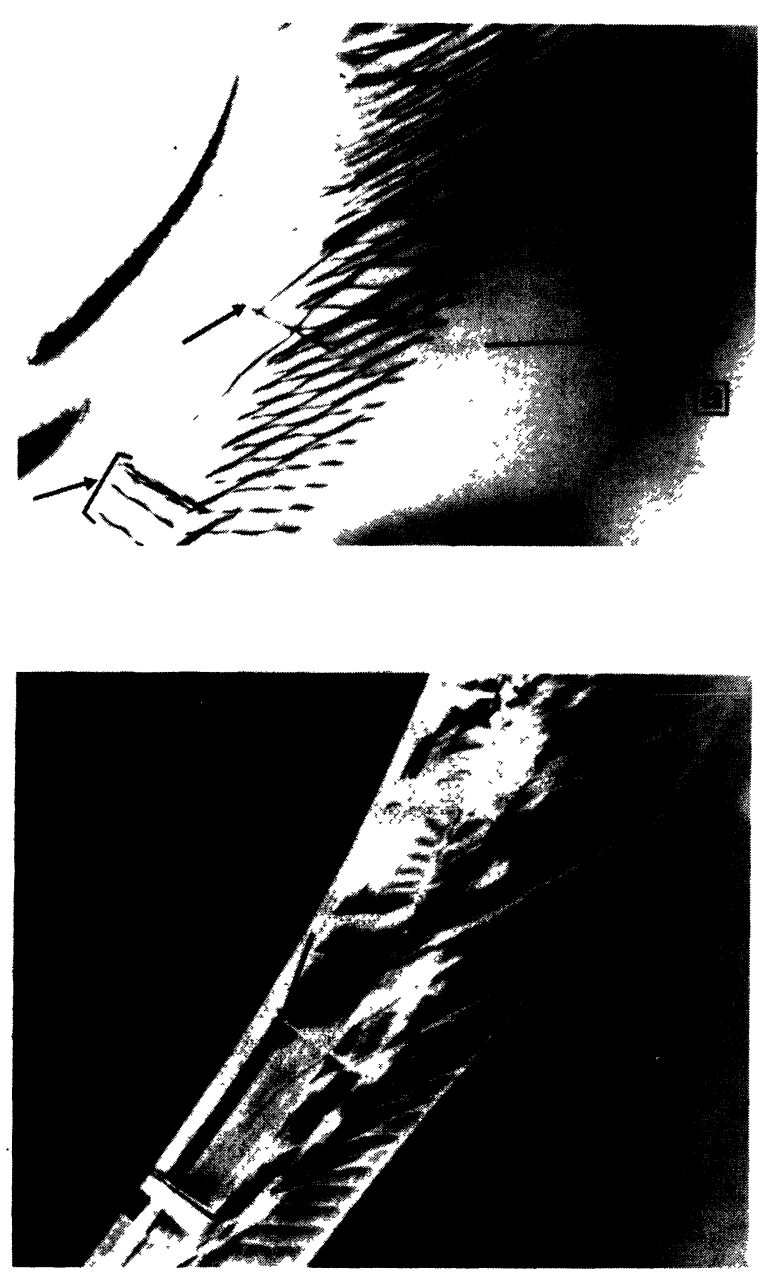

Fig. 5. - (111) microtwins. The screw partials with Burgers vector $1 / 6$ [121] are arrowed. The other partial dislocations are $30^{\circ}$ and screw dislocations. a $:$ Bright-field. $g=(220)_{\mathrm{M}}=(220)_{\mathrm{T}} \cdot \mathrm{b}:$ Dark-field. $\mathbf{g}=(11 \overline{1})_{\mathrm{T}}$. The matrix is out of contrast.

foils show that the perfect dislocations are emitted by the (154) abraded face (A. Lefebvre, unpublished). It can be concluded that as in In Sb, at higher temperatures [14], the deformation of

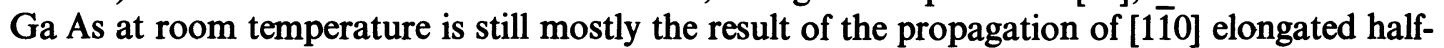
loop dislocations with very mobile $60^{\circ}(\alpha)$ fronts. On the contrary, the B glide bands contain stacking faults which should come from the (154) non-abraded face where sources are much fewer.

This interpretation is in keeping with the results of microindentation $[8,9]$ ! In the former reference, the asymmetry of the rosette dislocation patterns is attributed to the various mobilities of $\alpha$ and $\beta$ dislocations. In the latter reference, the TEM analysis of the same type of rosettes shows that one arm of the rosettes contains only perfect dislocations whereas the other arm contains stacking faults. We suggest the following interpretation for these results : the longer rosette arms should be due to the propagation of mobile perfect (or slightly dissociated) $\alpha$ dislocations while the shorter rosette arms should correspond to less mobile widely dissociated $\beta$ (or screw) dislocations. 
The wide dissociation of the $\beta$ (or screw) dislocations can be interpreted as follows. In the Ga As structure, there are six types of dissociated dislocations along $\langle 1 \overline{1} 0\rangle$ directions in the (111) plane as shown in figure 6. From [20], the $30^{\circ}(\beta)$ partial dislocation possesses the lowest mobility in all the partials and determines the mobility of the $\beta$ and screw dislocations. The lower mobility of the $30^{\circ}(\beta)$ partials could also account for the dissociation of the $\beta$ (or screw) dislocations by distinguishing the order of the constituent partials of these moving dislocations. When the $30^{\circ}(\beta)$ partial is the trailer, a widening of the stacking fault ribbon can be expected

\section{SCREW}

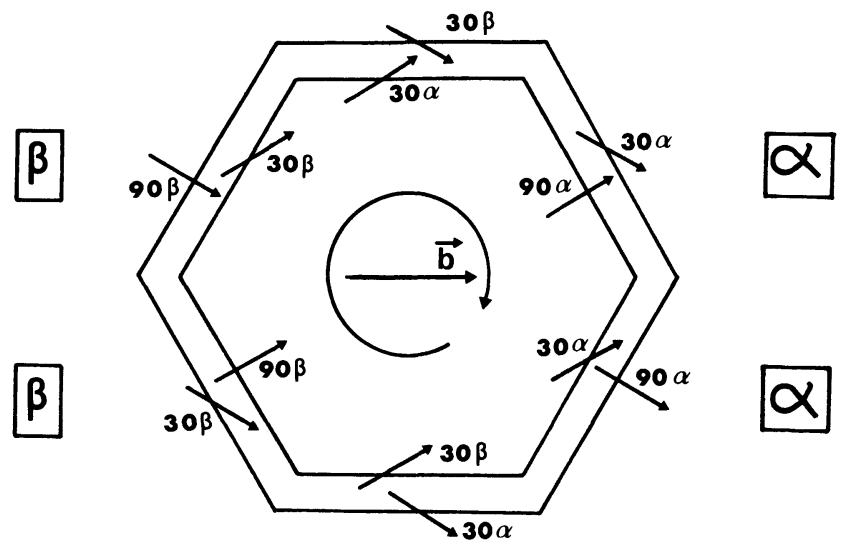

\section{SCREW}

Fig. 6. - The six types of dissociated dislocations along the $\langle 1 \overline{1} 0\rangle$ directions in the (111) slip plane.

if the applied stress is higher than the theoritical stress $\tau_{\mathrm{c}}$ required to overcome the back stress of the stacking fault. A rough estimate $\tau_{\mathrm{c}}$ is $\gamma_{\mathrm{SF}} / b \simeq 250 \mathrm{MPa}$ (with $\gamma_{\mathrm{SF}}=55 \mathrm{~mJ} / \mathrm{m}^{2}$ [21]); it is lower than the applied stresses in our experiments $(\simeq 750 \mathrm{MPa})$ and this is consistent with the large dissociations observed in the $\mathbf{B}$ glide bands. Further experiments are now being made to study the mobility of partial dislocations in Ga As deformed under high stresses.

\section{Acknowledgments.}

The authors wish to thank Mr. Bonnet (L.C.R.T. Thomson-Orsay-France) for providing the Ga As crystal.

\section{References}

[1] Steinhardt, H. and HaAsen, P., Phys. Status Solidi 49 (1978) 93.

[2] Mil'VidskiI, M. G., OsvenskiI, V. B. and Stolyarov, O. G., Neorg. Mater 1 (1965) 1898.

[3] Sazhin, N. P., Mil'vidskit, M. G., OsvenskiI. V. B. and Stolyarov, O. G., Sov. Phys. Solid State 8 (1966) 1539.

[4] Laister, D. and Jenkins, G. M., J. Mater. Science 8 (1973) 1539. 
[5] Karmouda, M., Thèse 3e cycle, Lille (1984).

[6] Alexander, H. and HaAsen, P., Solid State Phys. 22 (1968) 27.

[7] Wessel, K. and Alexander, H., Philos. Mag. 35 (1977) 1523.

[8] Warren, P. D., Pirouz, P. and Roberts, S. G., Philos. Mag. A 50 (1984) L 23.

[9] Hoche, H. R. and Schreiber, J., Phys. Status Solidi A 86 (1984) 229.

[10] Castaing, J., Veyssiere, P., Kubin, L. P. and Rabier, J., Philos. Mag. A 44 (1981) 1407.

[11] Rabier, J., Veyssiere, P. and Demenet, J. L., J. Physique Colloq. 44 (1983) C4-243.

[12] Ferre, D. and Farvacque, J. L., Phys. Status Solidi A 49 (1978) 737.

[13] Kesteloot, R., Thèse de 3e cycle, Lille (1981).

[14] Di Persio, J. and Kesteloot, R., J. Physique Colloq. 44 (1983) C4-469.

[15] Castaing, J., Cadoz, J. and Kirby, S. H., J. Am. Ceram. Soc. 64 (1981) 504.

[16] Howie, A. and Whelan, M. J., Proc. Roy. Soc. A 267 (1962) 206.

[17] Gomez, A. M. and Hirsch, P. B., Philos. Mag. A 38 (1978) 733.

[18] Clarebrough, L. M. and Forewood, C. F., Phys. Status Solidi 33 (1976) 355.

[19] Humble, P., Phys. Status Solidi 30 (1968) 183.

[20] Ninomiya, T., J. Physique Colloq. 40 (1979) C6-143.

[21] Gottschalk, H., Patzer, G. and Alexander, H., Phys. Status Solidi A 45 (1978) 207. 